Birkhäuser 

João Pedro Morais

Svetlin Georgiev

Wolfgang Sprößig

\section{Real Quaternionic Calculus Handbook}

Birkhäuser 
João Pedro Morais

CIDMA

University of Aveiro

Aveiro

Portugal

Wolfgang Sprößig

Institut für Angewandte Analysis

TU Bergakademie Freiberg

Freiberg

Germany
Svetlin Georgiev

Department of Differential Equations

University of Sofia St Kliment

Ohridski Faculty of Mathematics and Informatics

Sofia, Bulgaria

ISBN 978-3-0348-0621-3

DOI 10.1007/978-3-0348-0622-0

ISBN 978-3-0348-0622-0 (eBook)

Springer Basel Heidelberg New York Dordrecht London

Library of Congress Control Number: 2013957805

Mathematics Subject Classification 2010: 17C60, 30G35, 05A05, 15A66, 15A54, 51M05

(C) Springer Basel 2014

This work is subject to copyright. All rights are reserved by the Publisher, whether the whole or part of the material is concerned, specifically the rights of translation, reprinting, reuse of illustrations, recitation, broadcasting, reproduction on microfilms or in any other physical way, and transmission or information storage and retrieval, electronic adaptation, computer software, or by similar or dissimilar methodology now known or hereafter developed. Exempted from this legal reservation are brief excerpts in connection with reviews or scholarly analysis or material supplied specifically for the purpose of being entered and executed on a computer system, for exclusive use by the purchaser of the work. Duplication of this publication or parts thereof is permitted only under the provisions of the Copyright Law of the Publisher's location, in its current version, and permission for use must always be obtained from Springer. Permissions for use may be obtained through RightsLink at the Copyright Clearance Center. Violations are liable to prosecution under the respective Copyright Law.

The use of general descriptive names, registered names, trademarks, service marks, etc. in this publication does not imply, even in the absence of a specific statement, that such names are exempt from the relevant protective laws and regulations and therefore free for general use.

While the advice and information in this book are believed to be true and accurate at the date of publication, neither the authors nor the editors nor the publisher can accept any legal responsibility for any errors or omissions that may be made. The publisher makes no warranty, express or implied, with respect to the material contained herein.

Printed on acid-free paper

Springer Basel is part of Springer Science+Business Media (www.springer.com) 


\section{Preface}

Real Quaternionic Analysis is a multifaceted subject. Created to describe some phenomena in special relativity, electrodynamics, spin, etc. It has developed into a body of material that interacts with many branches of mathematics, such as complex analysis, harmonic analysis, differential geometry, differential equations, as well as into a ubiquitous factor in the description and elucidation of problems in mathematical physics. Quaternions have been towards Maxwell's equations. In the meantime, real quaternionic analysis has became a well-established branch of mathematics and greatly successful in many different directions. Quaternions have been successfully applied to signal processing, most notably pattern recognition. They can be used for image segmentation, finding structure based not only upon color, but repeating patterns. Quaternions may also be used to simplify derivations in computer vision and robotics, to develop computer applications in virtual reality, and so on.

This book is intended to provide material for an introductory one- or twosemester undergraduate course on some of the major aspects of real quaternionic analysis, with exercises. Alternatively, it may be used in a beginning graduate level course and as a reference. That it is why, rather than general theorems, we supply concrete examples and exercises which form the basis of this book. The exercises proposed at the end of each chapter are an essential part of it. The writing herein is straightforward and it is addressed to readers who have no prior knowledge of this subject and who have a basic graduate mathematics background: real and complex analysis, ordinary differential equations, partial differential equations and theory of distributions.

The detailed reference list proposed at the end is seen as an initial point for the development of the topics covered in the handbook. From a reader's point of view, we chose to present it at the end rather than throughout the text.

Here is a brief description of the topics covered in the first ten chapters.

Chapter 1 An introduction to and historical background on the discovery of the quaternions are provided. The definitions and general properties of quaternions are examined in detail.

Chapter 2 The notions of quaternion and spatial rotation are mastered. Some applications of quaternions to plane geometry are mentioned. 
Chapter 3 Studies sequences of quaternion numbers and their properties.

Chapter 4 Reviews the basic properties of quaternion power series and infinite products.

Chapter 5 The quaternion exponential, logarithmic and power functions are covered. A brief discussion on the notions of multiple-valued functions and branches is also presented.

Chapter 6 The quaternion trigonometric functions are defined.

Chapter 7 The quaternion hyperbolic functions are introduced.

Chapter 8 The main focus here is on the study of the inverses of the quaternion trigonometric and hyperbolic functions, and their properties.

Chapter 9 Matrices with quaternion entries are presented. In spite of the difficulties caused by the noncommutativity of the multiplication of quaternions, we still manage to introduce the concepts of determinant, rank, eigenvalues, and relations of similarity.

Chapter 10 Studies the concepts of monomials, polynomials and binomials involving quaternion numbers.

It is with great pleasure that we express our appreciation to all those who have expressed support, enthusiasm and encouragement in this adventure. We are forever indebted to our families and close friends for their patience, understanding and support: Lucília and Mário Morais, José António Morais, Ana Beatriz Pistola, Constança Sofia Morais, Francisco Elías, Laura, Borislav, Gerard and Nathalie, and Martina Sprößig. As regards to the present edition, our thanks go to Helmuth Malonek (Aveiro/Portugal), Isabel Cação (Aveiro/Portugal), Klaus Gürlebeck (Weimar/Germany), Tao Qian (Macau/China), Mahmoud Abul-Ez (Sohag/Egypt), Kou Kit Ian (Macau/China), Eckhard Hitzer (Fukui/Japan), Saburou Saitoh (Aveiro/Portugal), Hoai Le (Freiberg/Germany), and Inês Matos (Aveiro/Portugal) for helpful discussions and encouragement, and we especially thank the editor Thomas Hempfling (Birkhäuser) for the meticulous care with which he examined the entire manuscript. We are also grateful to the students who participated in the course "Function theory in higher dimensions" at the Technical University of Mining, Freiberg (Germany), whose enthusiasm, interest and dedication are admirable. For financial aid, the first named author wishes to express his gratitude to the Portuguese Foundation for Science and Technology ("FCT-Fundação para a Ciência e a Tecnologia”) via the postdoctoral grant SFRH/BPD/66342/2009. The first author's work is also supported by FEDER funds through COMPETEOperational Programme Factors of Competitiveness ("Programa Operacional Factores de Competitividade") and by Portuguese funds through the Center for Research and Development in Mathematics and Applications (University of Aveiro) and the FCT, within project PEst-C/MAT/UI4106/2011 with COMPETE number FCOMP-01-0124-FEDER-022690. 
Although the examples and exercises have been tested several times, we apologize in advance for any errors (typos) or just plain mistakes that you may find, and kindly ask you to bring them to our attention.

Aveiro, Portugal

João Pedro Morais

Sofia, Bulgaria

Svetlin Georgiev

Freiberg, Germany

Wolfgang Sprößig

November 2012 



\section{Contents}

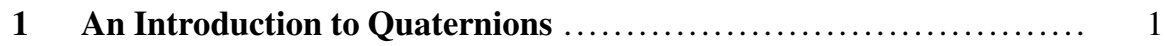

$1.1 \quad$ Basic Units ......................................... 3

$1.2 \quad$ Scalar and Vector Parts .................................. 4

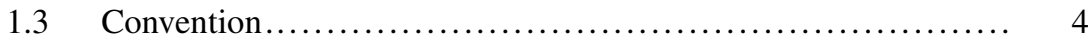

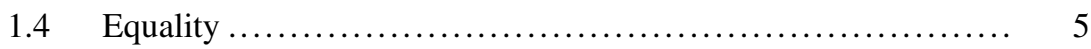

1.5 Arithmetic Operations................................. 5

$1.6 \quad$ Special Quaternions .................................... 7

1.7 Decomposition of Quaternions ............................ 8

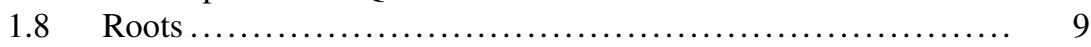

1.9 Quaternion Conjugation................................... 9

1.10 Quaternion Modulus and Quaternion Inverse ................. 10

1.11 Quaternion Quotient.................................... 11

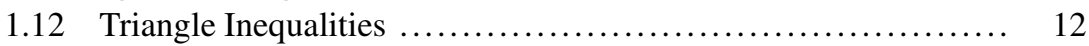

1.13 Quaternion Dot Product................................. 13

1.14 Quaternion Cross Product................................. 14

1.15 Mixed Product .......................................... 16

1.16 Development Formula.................................... 17

1.17 Sum Identity for the Double Vector Product .................. 17

1.18 Lagrange Identity ........................................ 18

1.19 Quaternion Outer and Even Products ....................... 19

1.20 Equivalent Quaternions ................................. 19

1.21 Polar Form of a Quaternion ............................... 22

1.22 Quaternion Sign and Quaternion Argument ................. 23

1.23 Quaternion Argument of a Product .......................... 24

1.24 Principal Argument ....................................... 24

1.25 de Moivre's Formula ................................... 24

1.26 Failure for Noninteger Powers ............................. 25

1.27 First Matrix Representation of Quaternions................... 26

1.28 Second Matrix Representation of Quaternions ................. 28

1.29 Advanced Practical Exercises............................... 29

2 Quaternions and Spatial Rotation ............................ 35

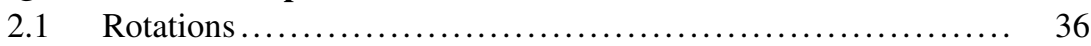

$2.2 \quad$ Composition of Rotations .................................. 39 
2.3 Rotation Matrix Representation ......................... 40

2.4 Quaternion Representation of Rotations ................... 42

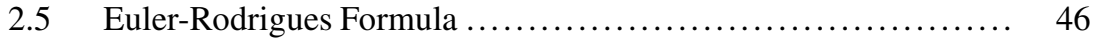

2.6 Applications to Plane Geometry ......................... 47

2.7 Advanced Practical Exercises............................ 49

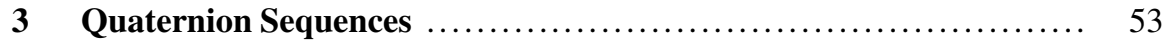

3.1 Quaternion Sequence.................................. 53

3.2 Scalar and Vector Parts of a Quaternion Sequence ............. 54

3.3 Symmetric, Conjugate, Modulus and Inverse of a Quaternion Sequence............................. 54

$3.4 \quad$ Arithmetic Operations.................................... 54

3.5 Convergence of a Quaternion Sequence...................... 56

3.6 Divergence of a Quaternion Sequence ..................... 56

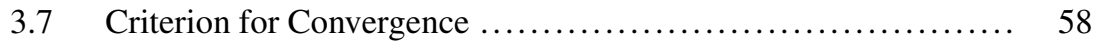

3.8 Certain 'Property Eventually' ........................... 59

3.9 Quaternion Subsequence ................................. 60

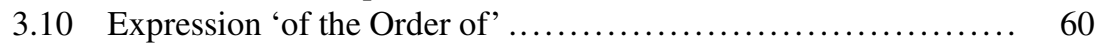

3.11 Equivalent Convergence Criteria .......................... 61

3.12 Cauchy Quaternion Sequence .......................... 61

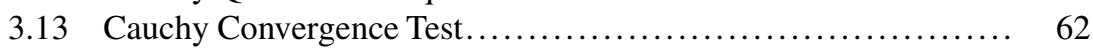

3.14 Summation by Parts .................................... 63

3.15 Abel Transformation ................................... 63

3.16 Advanced Practical Exercises............................ 64

4 Quaternion Series and Infinite Products ...................... 69

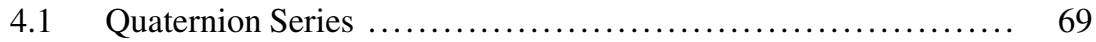

4.2 Arithmetic Properties of Quaternion Series ................... 70

4.3 Geometric Quaternion Series ........................... 70

4.4 A Necessary Condition for Convergence .................... 72

4.5 The $n$th Term Test for Divergence....................... 73

4.6 Absolute and Conditional Convergence of a Quaternion Series ... 73

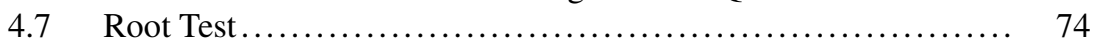

$4.8 \quad$ Ratio Test ................................................. 75

4.9 Quaternion Sequences of Bounded Variation .................. 76

4.10 Dirichlet's Test .......................................... 76

4.11 Alternating Series Test ................................. 77

4.12 Abel's Test........................................ 77

4.13 Quaternion Power Series ............................... 78

4.14 Radius of Convergence.................................. 79

4.15 Theoretical Radius ........................................ 81

4.16 The Arithmetic of Quaternion Power Series .................. 81

4.17 Infinite Products of Quaternion Numbers .................. 82

4.18 Absolute and Conditional Convergence of a Quaternion Product............................................. 82

4.19 Advanced Practical Exercises.......................... 83 
$5 \quad$ Exponents and Logarithms ................................. 87

$5.1 \quad$ Quaternion Natural Exponential Function .................... 88

5.2 Modulus, Argument, and Conjugate of the Quaternion

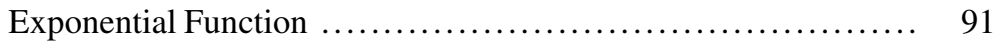

5.3 Quaternion Natural Logarithm Function ................... 92

5.4 Limits of the Quaternion Exponential and Logarithm Functions ........................................... 93

5.5 Principal Value of a Quaternion Natural Logarithm Function ..... 94

5.6 The Inverse of the Quaternion Natural Logarithm Function ...... 96

5.7 Quaternion Power Function........................... 97

5.8 General Properties of the Quaternion Power Function ........... 98

5.9 Principal Value of a Quaternion Power Function .............. 100

5.10 Advanced Practical Exercises............................. 100

6 Trigonometric Functions ................................... 107

6.1 Quaternion Sine and Cosine Functions..................... 107

6.2 Trigonometric Identities .................................. 109

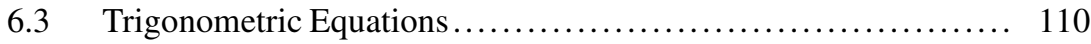

$6.4 \quad$ Zeros ............................................... 111

6.5 Quaternion Tangent and Secant Functions ................... 113

6.6 Quaternion Cotangent and Cosecant Functions................. 114

6.7 Advanced Practical Exercises........................... 115

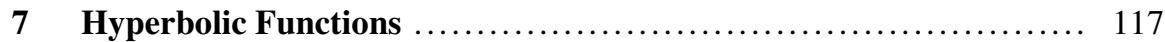

7.1 Quaternion Sine and Cosine Hyperbolic Functions ............. 117

7.2 Hyperbolic Identities ................................. 118

7.3 Equations with Hyperbolic Functions ....................... 120

7.4 Relation to Quaternion Sine and Cosine Functions .............. 121

$7.5 \quad$ Zeros ................................................. 121

7.6 Quaternion Tangent and Secant Functions .................. 122

7.7 Quaternion Cotangent and Cosecant Hyperbolic Functions....... 123

7.8 Advanced Practical Exercises............................. 123

8 Inverse Hyperbolic and Trigonometric Functions ................ 125

8.1 Quaternion Inverse Hyperbolic Sine and Cosine Functions ....... 125

8.2 Quaternion Inverse Hyperbolic Tangent Function ............... 129

8.3 Quaternion Inverse Trignometric Sine and Cosine Functions..... 130

8.4 Quaternion Inverse Trignometric Tangent Function ............ 130

8.5 Advanced Practical Exercises............................ 131

9 Quaternion Matrices ...................................... 133

9.1 Quaternion Matrices..................................... 133

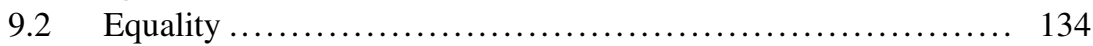

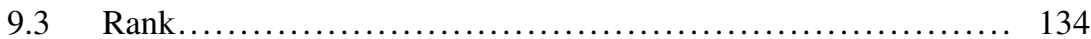

9.4 Matrix Arithmetic and Operations........................... 134

9.5 Special Quaternion Matrices ............................. 136

9.6 Basic Notions of Quaternion Matrices ..................... 137 
9.7 Inverse of a Quaternion Matrix ............................ 138

9.8 Quaternion Conjugate of a Matrix ....................... 139

9.9 Quaternion Transpose, Conjugate Transpose, and Inverse Matrices ................................ 140

9.10 Positive and Semi-positive Definite Quaternion Matrices ......... 141

9.11 Determinant of a Quaternion Matrix ...................... 141

9.12 Dieudonné Determinant................................... 143

9.13 Quaternion Eigenvalues and Eigenvectors .................. 144

9.14 Spectrum of a Quaternion Matrix ....................... 145

9.15 Right Eigenvalue Equalities ............................ 145

9.16 Advanced Practical Exercises............................ 146

10 Monomials, Polynomials and Binomials ...................... 149

10.1 Quaternion Monomials............................... 150

10.2 Quaternion Monomials Arithmetic and Operations ............. 151

10.3 Quaternion Polynomials ............................. 152

10.4 Factoring and Roots of Quaternion Polynomials ............... 152

10.5 Quaternion Binomial .................................. 154

10.6 Basic Relations of Quaternion Binomials ...................... 154

10.7 Inverse of the Quaternion Binomial ..................... 157

10.8 Basic Relations of Quaternion Inverse Binomials ............. 158

10.9 Advanced Practical Exercises............................. 162

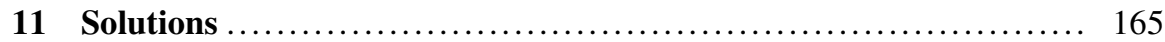

Bibliography $\ldots \ldots \ldots \ldots \ldots \ldots \ldots \ldots \ldots \ldots \ldots \ldots \ldots \ldots \ldots \ldots \ldots \ldots \ldots .211$

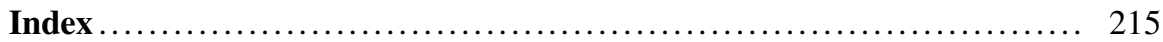

